\title{
Demandas sociales del sector educativo a la UPS
}

\author{
Nelson Raza Calderón*
}

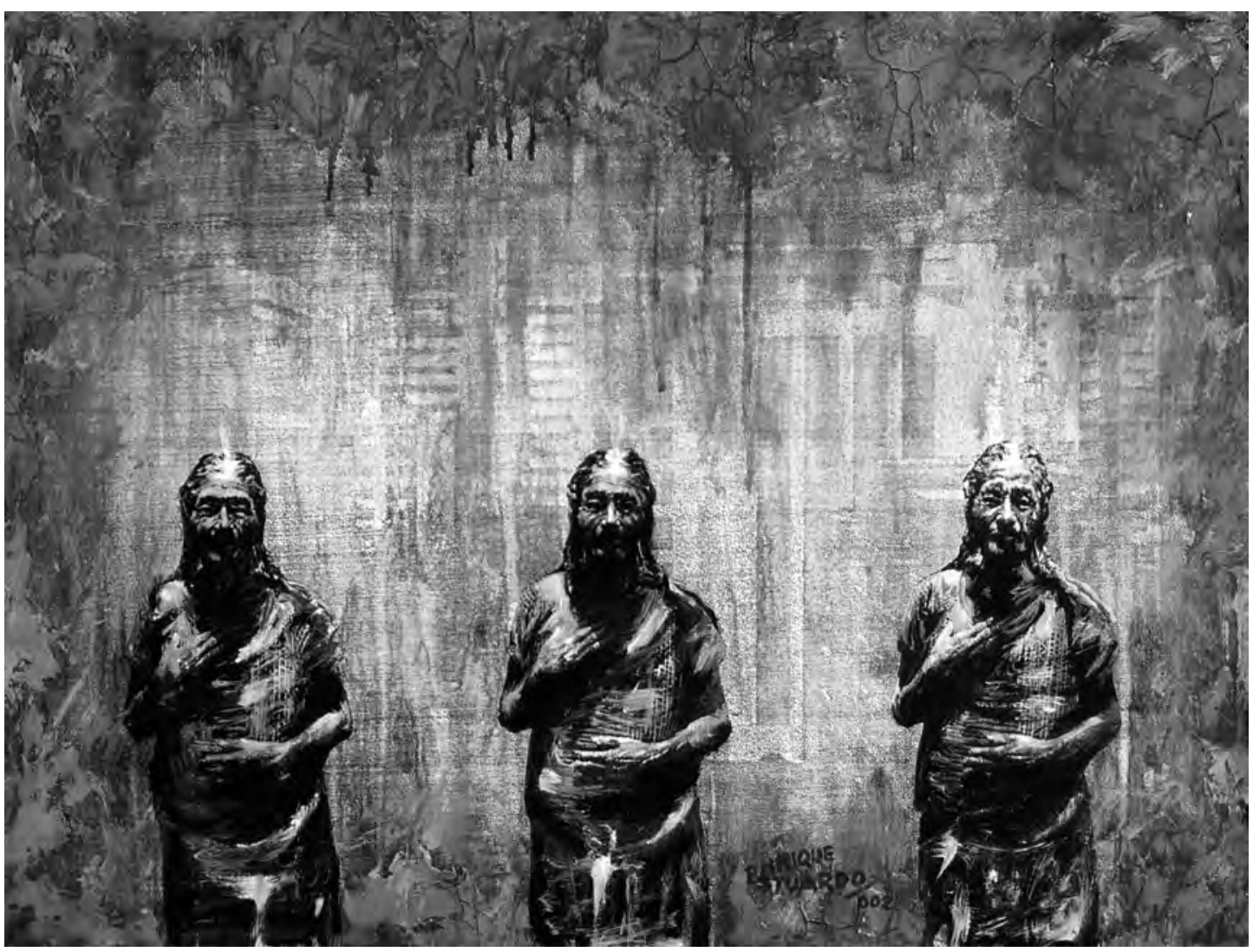

Urbana 2. Técnica mixta sobre tela. 2001

\section{Resumen}

La inteligencia, la creatividad, la formación en valores, los talentos y capacidades... pueden ser desarrollados en todos los niños y ni- ñas ecuatorianos través de un proceso educativo de calidad.

Partiendo de esta premisa, el propósito central de esta investigación es conocer si la gestión del docente de educación básica, es efectiva,

* Profesor principal de la UPS. Máster en Docencia. 
pertinente y en qué medida contribuye a mejorar la calidad educativa de este nivel.

En este trabajo se presentan los resultados de una investigación no experimental, transeccional exploratoria, en la que se entrevistaron a 115 docentes, de 85 planteles del Distrito Metropolitano de Quito.

Los resultados mostraron un nivel de gestión docente deficitario, el mismo que difícilmente puede contribuir a la formación integral de los educandos, peor aún a mejorar cualitativamente la oferta educativa de este nivel escolar.

La Carrera de Pedagogía de la UPS, toma los resultados obtenidos en este proceso para diseñar "Líneas de Investigación", con las que se buscará alternativas de solución a los complejos problemas de la educación básica y así mejorar la gestión de los docentes de este nivel.

\section{Introducción}

Aunque parezca una paradoja, ya que en los establecimientos educativos todavía se habla de las orientaciones de la última "reforma educativa", los docentes que desempeñan sus labores en la "educación básica", no pueden mejorar el nivel cualitativo de su accionar, porque desconocen, en la práctica los reales alcances de su gestión docente.

Por su lado, la Universidad también ha contemplado, a la distancia, sin inmutarse, los inquietantes resultados de la gestión docente en el nivel básico, que se desarrolla a partir de la mencionada "reforma" y que se avisoran a través de los magros resultados alcanzados en las últimas evaluaciones del rendimiento de los escolares.

La deficiente calidad de la educación básica es una constante que sigue permeando el sistema educativo nacional sin que se haya logrado la "formación integral" de nuestras niñas y niños. Y, en este contexto, la gestión del docente tiene un ineludible nivel de responsabilidad, porque es quien diseña, organiza, ejecuta y eva- lúa el proceso de enseñanza aprendizaje en el aula, que es un área de su exclusiva jurisdicción.

Para abordar el problema de la deficiente calidad de la educación básica, estimamos necesario formularnos una pregunta central: ¿Cuál es el nivel de efectividad y pertinencia, de la gestión del docente que labora en la educación básica, para impulsar un proceso de formación integral de calidad en los niños que asisten a la escuela?

Consecuentemente, el propósito fundamental de esta investigación, fue conocer si la gestión docente, en sus principales componentes, conduce a la formación integral de los educandos y mejorar la calidad de la educación, en el nivel básico.

La hipótesis que orientó el trabajo del equipo investigativo se estableció en los siguientes términos: "La gestión realizada por los docentes que laboran en los planteles de educación básica adolece de serias deficiencias, en sus principales áreas, que impiden o limitan ofrecer un servicio educativo de calidad con miras hacia la formación integral de los infantes".

Con estas inquietudes decidimos, con el respaldo de la Dirección de la Carrera de Pedagogía, de la UPS, realizar con la participación de las estudiantes, una investigación a 115 docentes, entre directores y/o profesores, de 85 escuelas ubicadas en el Distrito Metropolitano de Quito.

El proceso de enseñanza-aprendizaje de los estudiantes universitarios, debe estar anclado, en buena medida, en la resolución de problemas básicos de carácter profesional, según corresponda, a la naturaleza de su futuro ámbito de desempeño laboral. Por ello esta investigación es un espacio también de aprendizaje.

Nuestros resultados confirmaron, que el nivel de gestión docente es deficitario. Se presentan: limitaciones, desconocimiento, omisiones, incomunicación, descoordinación... en la gestión, que inevitablemente impactan en el proceso de formación de los niños y niñas y que difícilmente contribuyen a elevar la calidad de la educación básica. 


\section{Materiales y métodos}

El enfoque metodológico que orientó el desarrollo de este ejercicio investigativo fue, básicamente, no experimental, transeccional, multimodal.

El alcance de esta investigación es de carácter exploratorio. Sus resultados, por consiguiente, deberían ser ampliados, confirmados y profundizados, con investigaciones de mayor alcance. No obstante, sus resultados pueden constituirse en posibles hipótesis que orienten y alienten trabajos de mayor profundidad y estructuración, mediante la identificación de Líneas de Investigación, para los estudiantes de la Carrera.

La técnica que se utilizó para la recolección de datos e información fue la entrevista en profundidad, en la que se utilizó una guía-cuestionario.

El universo de la investigación estuvo constituido por un grupo aleatorio de 85 planteles de educación básica ubicados, principalmente, en el Distrito Metropolitano de Quito, en los sectores de residencia de las estudiantes, en consideración del conocimiento de las zonas geográficas seleccionadas.

En cada establecimiento educativo se buscó como sujeto informante al Director/a y/o docente en ejercicio. Este procedimiento permitió establecer una muestra de de 115 docentes investigados.

\section{Resultados}

\section{Profesores especializados por áreas}

Las áreas de Matemáticas y Lenguaje, se presentan con un mayor porcentaje de profesores especializados que laboran en sus respectivos campos del conocimiento, le siguen las ciencias sociales y naturales. Además se constató que, en las instituciones investigadas, existen maestros que tienen otras especialidades como: inglés, educación física, música y artes plásticas, que abarcan el $24 \%$.
Cuadro 1. Profesores especializados por áreas

\begin{tabular}{|l|c|c|}
\hline MATERIA & FRECUENCIA & $\%$ \\
\hline Lenguaje & 62 & 19,74 \\
\hline Ciencias Sociales & 57 & 18,15 \\
\hline Matemáticas & 63 & 20,06 \\
\hline Ciencias Naturales & 58 & 18,47 \\
\hline Otros & 74 & 23,56 \\
\hline Total & 314 & 100 \\
\hline
\end{tabular}

Esta información nos permite apreciar que existe, inicialmente, un intento por trabajar en la escuela por áreas de especialización del conocimiento. Este ensayo, para el autor de estas líneas, debe consolidarse, ya que podría coadyuvar a mejorar el nivel cualitativo de la educación, con el impulso de un sistema curricular educativo innovador fundamentado en la formación por competencias, que desarrolle en la práctica un conjunto de capacidades investigativas tanto en docentes como en estudiantes con el propósito de generar procesos pertinentes de construcción y reconstrucción de sus propios saberes (Robayo et. al, 2006: 2).

En este contexto, la formación y preparación pertinente de los docentes responsables de estas áreas, por parte de la Universidad es un desafío que debe ser asumido con oportunidad, para cumplir con su responsabilidad social.

\section{Modelos educativos y misión institucional}

De acuerdo con la información proporcionada parece no existir una comprensión cabal del sentido de Modelo Educativo. Este hecho es muy inquietante.

No obstante, se llega a establecer en esta investigación, con no pocas dificultades, que el modelo educativo que actualmente se utiliza en mayor proporción en las instituciones educativas es el constructivista con un $73 \%$. 
Cuadro 2. Modelos educativos

\begin{tabular}{|l|c|c|}
\hline MODELO & FRECUENCIA & $\%$ \\
\hline Constructivista & 84 & 73,04 \\
\hline Conductista & 4 & 3,34 \\
\hline Holístico & 3 & 2,60 \\
\hline $\begin{array}{l}\text { Otros: } \\
\text { Cultural, social }\end{array}$ & 24 & 20,86 \\
\hline Total & 115 & 100 \\
\hline
\end{tabular}

La aplicación de este modelo ubica al alumno en el centro del proceso de enseñanza-aprendizaje; se impulsa a los niños a ser los actores de la generación de su propio conocimiento. Novack -en este contexto-, concibe al aprendizaje como el proceso de construcción del conocimiento, con una directa participación de las capacidades cognitivas del estudiante, así como sus intereses y sus propios elementos culturales lo que le permite apropiarse del conocimiento construido en una fase inicial, para luego una etapa progresiva arrancar su formación como ser humano con la construcción y reconstrucción de otros conocimientos. En esta fase ulterior el niño, por exigencias de la naturaleza del propio proceso constructivista, desarrolla una serie de competencias individuales y sociales que surgen de la relación con los demás miembros de su entorno

Es interesante anotar que, un modelo constructivista, conlleva en su concepción matricial una voluntad positiva, que se traduce en una actitud favorable de "querer aprender" y que para ello tiene ciertos principios y finalidades que lo orientan, hacia "la adaptación, transformación, desarrollo, individual y grupal, formación para el trabajo y en definitiva formación para la vida" (Robayo et. al, 2006: 7).

La aplicación práctica de este modelo debería producir resultados alentadores en la educación de los infantes, lamentablemente, por la naturaleza propia de esta investigación, no con- tamos con las suficientes evidencias empíricas que avalicen sus resultados, los mismos que bien merecerían ser conocidos con mayor amplitud, con una investigación de mayor alcance.

Desde luego, también coexisten otros modelos, como el conductista y holístico, que manejan los docentes, en menor proporción.

\section{Misión y propósito}

La misión es la "razón de ser de la institución" que todos los docentes de un establecimiento educativo deberían conocer y más que ello, realizar sus mejores esfuerzos para tratar de alcanzar su concreción de manera colectiva, trabajando en equipo, con la cohesión y orientación requeridas.

Cuadro 3. Percepciones de la misión y propósito

\begin{tabular}{|l|c|c|}
\hline Rangos & FRECUENCIA & $\%$ \\
\hline $\begin{array}{l}\text { Formar al educando } \\
\text { de manera integral. }\end{array}$ & 35 & 30,43 \\
\hline Educación de calidad. & 20 & 17,4 \\
\hline $\begin{array}{l}\text { Fomentando los } \\
\text { valores cristianos. }\end{array}$ & 10 & 8,7 \\
\hline No conoce. & 50 & 43,47 \\
\hline TOTAL & 115 & 100 \\
\hline
\end{tabular}

En este punto surge una paradoja en la investigación: la mayoría de los docentes expresan conocer la Misión de su institución en un $99,13 \%$. En principio, esto sería muy importante, ya que permitiría realizar la tarea del cuerpo docente con una estrategia y un horizonte definidos. No obstante, al solicitarse el propósito esencial de la Misión un 44,6\% no tiene claro su contenido, o sea el conocimiento de la Misión se reduciría al 54,53\%. Como se puede apreciar existe una contradicción muy clara difícil de ser explicada en estas líneas. 
Cuadro 4. Percepciones de la misión institucional

\begin{tabular}{|l|c|c|}
\hline Opción & FRECUENCIA & $\%$ \\
\hline Conoce & 114 & 99,13 \\
\hline No Conoce & 1 & 0,87 \\
\hline Total & 115 & 100 \\
\hline
\end{tabular}

Por su lado, los docentes consultados responden que la finalidad de la Misión institucional es la formación integral del niño en un $29,5 \%$; brindar una educación de calidad un $17,4 \%$, fomentar los valores cristianos en un $8,7 \%$ y un $44,6 \%$ no la conocen, tal como ya se afirmó (supra).

Sería conveniente, por parte de UPS, profundizar el conocimiento de este aspecto mediante investigaciones de mayor alcance, ya que somos muy conscientes de que quedan algunos vacíos que merecerían un estudio estructurado de carácter explicativo.

\section{Visión institucional}

La Visión, en la perspectiva del pensamiento estratégico administrativo, es el "sueño que debe ser alcanzado" en un tiempo determinado, con el esfuerzo permanente, constante y proactivo de todos los integrantes de la comunidad educativa, dirigidos por una instancia de liderazgo situacional con un alto poder de efectividad.

Cuadro 5. Visión institucional

\begin{tabular}{|l|c|c|}
\hline OPCIÓN & FRECUENCIA & $\%$ \\
\hline Conoce & 75 & $65,2 \%$ \\
\hline No conoce & 40 & $34,8 \%$ \\
\hline Total & 115 & 100 \\
\hline
\end{tabular}

Necesario es reconocer, desde luego, que esta instancia debe lograr un empoderamiento del contenido de la Visión Institucional, median- te un proceso de comunicación asertivo, que motive a cada sector del establecimiento educativo a alcanzar los objetivos institucionales asignados, en un contexto de calidad, oportunidad, pertinencia y alto rendimiento.

Cuadro 6. Variables de la visión institucional

\begin{tabular}{|l|c|c|}
\hline RANGOS & FRECUENCIA & $\%$ \\
\hline $\begin{array}{l}\text { Formar alumnos capaces } \\
\text { y seguros }\end{array}$ & 59 & 79,73 \\
\hline $\begin{array}{l}\text { Posicionamiento } \\
\text { institucional }\end{array}$ & 11 & 14,86 \\
\hline Docencia eficiente & 5 & 5,41 \\
\hline Total & 75 & 100 \\
\hline
\end{tabular}

Al respecto en nuestra investigación, el 65\% manifiesta conocer la Visión de la institución; y, un significativo 34,8\% desconoce. Según este último dato, un considerable grupo de docentes realiza su gestión sin un propósito definido de largo alcance. Sus esfuerzos van en múltiples direcciones sin que exista un sueño por conseguir que guíe, aunque sea, de manera primaria, su gestión.

En las instituciones que conocen la Visión, ésta va enfocada a la formación del alumno en un $79,73 \%$; en un $14 \%$ a la necesidad de que su institución sea reconocida y aprobada y en un $5 \%$ que cada docente logre excelencia en la realización de su labor.

Ante esta situación es necesario advertir, que es vital que las instituciones logren cumplir lo que han planteado, tanto en su misión como en su visión, para que la educación pueda logre desarrollar los talentos diversos y las capacidades de los estudiantes.

\section{Limitaciones de gestión administrativa}

Uno de los problemas más frecuentes que se presenta es la falta de recursos económicos en un $19 \%$, para afrontar diversidad de problemas

No obstante, lo detectado en otros: $44 \%$, 
es lo más preocupante, ya que la falta de comunicación, la poca o ninguna colaboración de los distintos segmentos de la comunidad educativa, junto al retraso en el pago a los maestros, constituyen serios problemas que inciden de diversa manera en la gestión técnico-pedagógica y admi- nistrativa del establecimiento educativo. Estos factores, sin duda, provocan corto circuitos que detienen, desvían, deterioran y neutralizan la capacidad de logro institucional en las diversas esferas de gestión de un establecimiento educativo.

Cuadro 7. Gestión administrativa

\begin{tabular}{|l|c|c|}
\hline RANGOS & FRECUENCIA & $\%$ \\
\hline INFRAESTRUCTURA & 14 & 12,17 \\
\hline RECURSOS ECONÓMICOS & 22 & 19,13 \\
\hline PERSONAL ADECUADO & 19 & 16,52 \\
\hline NINGUNO & 16 & 13,91 \\
\hline $\begin{array}{l}\text { OTROS } \\
\text { Impuntualidad en los pagos } \\
\quad \text { a los maestros. } \\
\quad \text { Falta de comunicación. }\end{array}$ & 44 & 38,26 \\
\hline Falta de colaboración & & 100 \\
\hline TOTAL & 115 & \\
\hline
\end{tabular}

\section{Capacitación a los docentes}

Los docentes reciben capacitación, fundamentalmente sobre aspectos metodológicos referidos al cómo realizar la planificación exigida en la "reforma curricular", planteada varios años atrás, sin que haya logrado consolidarse. Pero la educación es un campo en el que surgen, de manera permanente, nuevos cambios que la dinamizan y que necesitan ser conocidos. Por ello, sin dejar de lado su preparación básica profesional, es importante su actualización permanente, sobre la base de un estudio/diagnóstico de las necesidades y principales requerimientos.
Cuadro 8. Opciones de capacitaciones

\begin{tabular}{|l|c|c|}
\hline OPCIONES & FRECUENCIA & $\%$ \\
\hline Sí & 80 & 69,56 \\
\hline No & 35 & 30,44 \\
\hline Total & 115 & 100 \\
\hline
\end{tabular}

Finalmente es necesario acotar que la Universidad tiene, en la época actual, un serio desafío para formar y capacitar a los docentes que, en su calidad de directivos institucionales, sean capaces de gerenciar y ejecutar proyectos 
estratégicos de desarrollo educativo, tendientes a formar personas que, en base a una sólida preparación ética, altamente competente y plural, puedan afrontar con éxito contextos sumamente cambiantes, de alta diversidad y complejidad cultural, científica y tecnológica, con miras hacia la conformación de sociedades más justas y equitativas.

\section{Fuentes para la elaboración del cu- rrículo}

El Currículo es el instrumento que sirve de base para orientar el desarrollo de la gestión del docente, por ello es de trascendental importancia conocer cuáles son las fuentes, objetivos, recursos didáctico que han sido conside- rados por los maestros para ejecutar sus tareas cotidianas.

Sobre este particular, los docentes investigados reconocen que las fuentes más utilizadas para la construcción del Currículo son: a) las que se producen a en la reforma interna de cada institución en un $40 \%$; b) la reforma curricular del Estado en $29,56 \%$; y c) las habilidades y destrezas de cada docente en un 30,44\%.

Si tomamos en consideración las reflexiones realizadas en lo referente a la misión, visión y limitaciones administrativas (supra) las respuestas dadas por los maestros, tienden a producir mayores incertidumbres que certezas. Quizá, entonces los elementos que proporciona la "reforma curricular" impulsada por el Estado sea la fuente curricular de mayor consistencia.

Cuadro 9. Fuentes para la construcción del currículo

\begin{tabular}{|c|c|c|}
\hline RANGOS & FRECUENCIA & $\%$ \\
\hline Reforma Curricular & 34 & 29,56 \\
\hline $\begin{array}{l}\text { Reforma Interna } \\
\text { Talleres } \\
\text { - } \quad \text { Macro y Micro planificaciones } \\
\text { - } \quad \text { Aplicación de proyectos de beneficio } \\
\quad \text { para el desarrollo de la educación. }\end{array}$ & 46 & 40 \\
\hline Habilidades y destrezas del docente & 35 & 30,44 \\
\hline Total & 115 & 100 \\
\hline
\end{tabular}

Este es un aspecto prioritario que debe ser profundizado ya que cada una de las respuestas consignadas requiere ser investigada con mayor amplitud dada su trascendencia en el campo educativo.

\section{Principales objetivos del currículo}

La "formación integral”, como categoría científica en el campo de la educación, tiene como punto de referencia central el currículo, con sus fines y objetivos muy claros y precisos por alcanzar. Y éstos deben abarcar al desarrollo de todas las capacidades y potencialidades del estudiante de manera global. Es muy ilustrativa la reflexión de Reinaldo Suárez, al respecto, cuando expresa: "Las potencialidades humanas son de orden físico, mental, emocional, social, estético, político y ético. Es por tanto un error reducir la actividad educativa y escolar exclusiva o prevalentemente a la transmisión de conocimientos...". 
Cuadro 10. Objetivos del currículo

\begin{tabular}{|l|c|c|}
\hline RANGOS & FRECUENCIA & $\%$ \\
\hline Progreso del educando: & & \\
\hline - Adaptación del niño en el aula. & & \\
\hline- Mayores exigencias & 55 & 52 \\
\hline- Cumplimiento del currículo & 1 & 0,94 \\
\hline Progreso de la comunidad: & & \\
\hline Conocimiento: & 22 & 19,13 \\
- Formar con buenas bases al niño & & 24,34 \\
- Mejorar la calidad de aprendizaje & 28 & 8,59 \\
\hline Destrezas: & 9 & 100 \\
\hline - Mejorar la calidad de procesos & 115 & \\
\hline Total & & \\
\hline
\end{tabular}

En nuestro caso, las repuestas formuladas por casi la mitad de los sujetos investigados respecto a los objetivos que se trata de alcanzar con la ejecución del Currículo son demasiado ambiguas. Veamos: lograr el progreso del educando, en términos muy generales, plantea un $52 \%$; el desarrollo de sus destrezas, sin especificar cuáles, 24\%; y, lograr conocimientos un $19,13 \%$.

Las ambigüedad de estas respuestas no permiten apreciar que es lo que realmente estamos tratando de alcanzar en y con la formación de los niños a través de la ejecución del currículo, por parte de los docentes en estos establecimientos educativos.

\section{Recursos didácticos utilizados en la institución}

El proceso docente involucra un conjunto lógico y coherente de formas de aprender y organizar el aprendizaje que abarca los contenidos, métodos, recursos didàcticos, tiempos, se- cuencias de conformidad con la naturaleza y complejidad del conocimiento que se trate de abordar.

Desde este ángulo, en la época actual, es importante visualizar el impacto de las innovaciones tecnológicas en la sociedad y de manera específica en la calidad de la docencia. En este contexto merece un particular análisis el uso de las nuevas tecnologías de la información y la comunicación (NTICs).

\section{Cuadro 11. Recursos educativos}

\begin{tabular}{|l|c|c|}
\hline RECURSOS & FRECUENCIA & $\%$ \\
\hline Textos & 28 & 24,36 \\
\hline Material impreso & 16 & 13,91 \\
\hline Digital & 7 & 6,08 \\
\hline Computación & 14 & 12,17 \\
\hline Audiovisuales & 10 & 8,69 \\
\hline Internet & 26 & 22,60 \\
\hline Otros & 14 & 12,07 \\
\hline Total & 115 & 100 \\
\hline
\end{tabular}


Al decir de Angelo Benvenuto "Las TIC son un recurso poderoso, cuya incorporación debe considerar nuevos roles del profesor, nuevos contenidos, nuevos programas y nuevas formas de evaluar ya que por si mismas las TIC e Internet no educan ni reemplazan al profesor; su incorporación exige revisar sistémica, corporativa y transversalmente los actuales contextos educativos"

El uso de las NTICs optimizan la gestión de los procesos de aprendizaje ya que involucran un conjunto convergente de tecnologías que posibilitan la "adquisición, producción, almacenamiento, tratamiento, comunicación, registro, acceso y presentación de datos, información y contenidos, en forma alfanumèrica, imàgenes, videos, sonidos..." (Benvenuto, 2006: 2) y estos son los insumos básicos del conocimiento.

Para el autor de estas reflexiones, los sistemas educativos contemporáneos, en nuestros entornos sociales no pueden bajo ningún precepto ideológico, étnico, religioso, cultural... convertirse en gethos y aislarse del avance y de las innovaciones científico-tecnológicas.
Los docentes consultados sobre este tópico dejan traslucir una realidad inquietante. Si bien utilizan diversidad de recursos didácticos para apoyar su gestión en el aula, tal como se puede apreciar en la tabla precedente, la mayoría de estos materiales, en un $44 \%$, son de carácter tradicional. Hay una tendencia incipiente hacia la búsqueda de otros recursos que se inscriben en las nuevas tecnologías de la información (NTICs) que merecerían el soporte tecnológico por parte de la UPS.

\section{Estrategias de enseñanza - aprendi- zaje}

Las estrategias de aprendizaje-enseñanza, en la actualidad deben ser consideradas, como mecanismos muy estructurados que conllevan un alto sentido de motivación para promover en el estudiante, desde todas las disciplinas, el desarrollo de la inteligencia, la creatividad y la formación de valores humanos (Cortijo, 2006: 115).

Cuadro 12. Estrategias de enseñanza - aprendizaje

\begin{tabular}{|l|c|c|}
\hline RANGOS & FRECUENCIA & $\%$ \\
\hline $\begin{array}{l}\text { Juegos didácticos: } \\
\text { - Trabajo dinámico en el patio o aula }\end{array}$ & 35 & 24,47 \\
\hline $\begin{array}{l}\text { Investigación: } \\
\text { - Proyectos }\end{array}$ & 22 & 19,13 \\
\hline $\begin{array}{l}\text { Ejercicios de socialización: } \\
\text { - Socialización }\end{array}$ & & \\
- Globalización & 18 & 16,4 \\
\hline Ejercicios Prácticos & & \\
\hline $\begin{array}{l}\text { Otras: } \\
\text { - Lúdicas }\end{array}$ & & 46 \\
\hline Eclécticas & 115 & 100 \\
\hline Total & & \\
\hline
\end{tabular}


La consideración estratégica de esta triada: valores-inteligencia-creatividad por los sistemas educativos contemporáneos es, sin lugar a duda, el sustento cultural para que los estudiantes puedan navegar, con ciertas garantías, en la compleja, desafiante y contradictoria sociedad del conocimiento y la información, con posibilidades de acceso a la construcción de nuevos saberes y conocimientos. El saber cambia al mundo, y nuestro mundo esta cambiando con la prontitud de los saberes nuevos. Todos sentimos que se abrió la puerta aunque no sepamos a dónde conduce. Es como otro big-bang. El tiempo ahora es más corto y el espacio es más pequeño: lo uno se le denomina "acelereción de la historia”, lo otro es la aldea global. Son las dimensiones objetivas $y$, en especial, las dimensiones subjetivas de la nueva sociedad, la sociedad del conocimiento" (Gómez Buendía, 1999: 2).

En este escenario las estrategias de aprendizaje juegan un papel fundamental en el desarrollo de las competencias cognitivas, procedimentales y afectivas del estudiante. Los datos recogidos en la investigación arrojan resultados poco alentadores: las estrategias de enseñanzaaprendizaje más utilizadas son: las lúdicas, y eclécticas en un porcentaje de $46 \%$ y los juegos didácticos con un $24,47 \%$. No hay una clara distinción entre lúdicas y juegos didácticos que constituyen las técnicas básicas de los procesos de aprendizaje.

Un porcentaje reducido del 19,3\% toma en cuenta a la investigación, mediante el desarrollo de pequeños proyectos como estrategias de aprendizaje. Esto deja al estudiante en una gran desventaja en la sociedad del conocimiento en la que... el valor agregado ya no proviene de los factores clásicos de la producción: tierra capital y trabajo: viene de la tecnología, antes que nada. Por eso se han agigantado la productividad humana y la retribución al saber"( Gómez, 199: 3)

Este es un aspecto de primordial importancia para la formación de los estudiantes de la educación básica que, sin embargo por la información recolectada, no tiene mayor trascendencia para los docentes de este nivel.

\section{Evaluación del aprendizaje de los niños}

En todo tipo de organización ya sea de carácter social, empresarial, pública o privada, es indispensable evaluar los resultados del trabajo realizado, con la finalidad de conocer los resulta-

Cuadro 13. Variables de evaluación

\begin{tabular}{|l|c|c|}
\hline ¿Qué se evalúa? & FRECUENCIAS & $\%$ \\
\hline Destrezas & 16 & 13,91 \\
\hline Conocimientos & 80 & 69,56 \\
\hline Destrezas y conocimientos. & 19 & 16,53 \\
& 115 & 100 \\
\hline Pruebas de evaluación: & & 56,52 \\
- Exámenes/Pruebas & 65 & 16,52 \\
- Trabajos en clase y en la casa & 19 & 8,69 \\
- Investigaciones & 10 & 4,36 \\
- Exposiciones & 5 & 13,91 \\
\hline Actuación en clase & 16 & 100 \\
\hline Total & 115 & \\
\hline
\end{tabular}


dos de la gestión, reorientar el desarrollo de las acciones, reformular objetivos, métodos y procedimientos. Este mecanismo permite tomar las decisiones pertinentes, adecuadas y oportunas para mejorar los procesos de gestión.

"El proceso de evaluación dentro de una institución educativa cobra una importancia capital ya que se trabaja en función de la transformación de seres humanos, donde los errores hay que corregirlos de inmediato..." (Cortijo, 2006: 133).

La evaluación del aprendizaje en los planteles consultados deja muchas dudas e inquietudes. En primer término, se detecta que se evalúa únicamente conocimientos y destrezas. Solamente conocimientos un 69,56\%; destrezas y conocimientos un $16,53 \%$ y solamente destrezas un $13 \%$. Como se puede advertir no se evalúa ninguna otra dimensión del desarrollo humano de los infantes, a pesar de que casi siempre está presente la premisa de la "formación integral" de los niños, tal como se pudo apreciar el criterio mayoritario de los docentes en el contenido de la "Misión institucional".
En cuanto a los procedimientos y mecanismos se conoce que la evaluación se efectúa, por parte de los docentes, utilizando las clásicas pruebas y exámenes convencionales en un $56,52 \%$; los trabajos en clase y en la casa en un $16,53 \%$. Y tal como se ya manifestó anteriormente, la investigación, como eje formativo-evaluativo apenas cuenta con un $8,69 \%$, con sus correspondientes consecuencias.

\section{Evaluación del trabajo docente}

La evaluación del trabajo docente es, sin duda, uno de los aspectos de mayor relevancia para comprender el nivel de logro, las limitaciones y en muchas ocasiones los fracasos de la gestión del maestro. Sus resultados deben servir para tomar las decisiones más conducentes al mismo maestro y a los directivos en las diferentes áreas de su gestión: propuesta curricular, contenidos, metodologías, recursos, estrategias...

Cuadro 14. Evaluación del trabajo docente

\begin{tabular}{|l|c|c|}
\hline OPCIÓN & FRECUENCIA & $\%$ \\
\hline SÍ & 98 & 85,21 \\
\hline NO & 17 & 14,79 \\
\hline & 115 & 100 \\
\hline
\end{tabular}

Cuadro 15. Mecanismo de evaluación

\begin{tabular}{|l|c|c|}
\hline RANGOS & FRECUENCIA & $\%$ \\
\hline VISITAS & & \\
- Del Director & 63 & 54,78 \\
\hline Supervisor & & \\
\hline MECANISMOS & & \\
- Planif. de la institución & & \\
- Planif. del maestro & 28 & 24,36 \\
\hline
\end{tabular}




\begin{tabular}{|l|c|c|}
\hline RANGOS & FRECUENCIA & $\%$ \\
\hline $\begin{array}{l}\text { TRABAJO DE AULA } \\
\text { En el momento de enseñar } \\
\quad \text { a los niños }\end{array}$ & & \\
\hline $\begin{array}{l}\text { OTRAS FORMAS } \\
\text { Mediante la evaluación a los alumnos }\end{array}$ & & 6,08 \\
\hline$\quad$ Las sesiones de los padres & 17 & 14,78 \\
\hline TOTAL & 115 & 100 \\
\hline
\end{tabular}

En el caso que nos ocupa, en primer término, es interesante conocer que se realiza la evaluación del trabajo de los docentes en un significativo porcentaje: el $85,21 \%$. Falta un $14,79 \%$ que está ausente de este proceso.

En segundo término, los mecanismos que se utilizan para evaluar el desempeño del docente son diversos. El Director de la institución manifiesta, en un $54,78 \%$, que es muy importante evaluar al docente mediante visitas al aula donde se imparte el conocimiento; mediante los planes de trabajo del maestro en un 24,36 ; otras formas en un $14,78 \%$ y finalmente trabajo de aula en un $6,08 \%$.

Finalmente, es necesario reconocer que la evaluación del desempeño de la gestión de los docentes deberá tener un vínculo de articulación directa con el sistema de desarrollo del talento humano institucional: selección, promoción, capacitación, reconocimiento..., para cumplir con los fines de mejoramiento permanente de la calidad de los procesos del establecimiento educativo.

\section{Conclusiones}

- La investigación permitió conocer el bajo nivel de efectividad y pertinencia de la gestión del docente de la Educación Básica y su escaso y a veces nulo aporte al mejoramiento cualitativo de la oferta educativa. Es necesario, sin duda, profundizar estos conocimientos mediante un proceso investigativo de mayor estructuración institucional, por parte de la Carrera de Pedagogía de la UPS-Q, para posibilitar la búsqueda de respuestas y posibles oportunidades de mejoramiento.

- Existe la tendencia, en la mayoría de los establecimientos educativos, al trabajo docente especializado por áreas del conocimiento. Sería interesante y oportuno estudiar la posibilidad de responder a este requerimiento, por parte de la Carrera de Pedagogía de la UPS, considerando no sólo las tradicionales áreas del lenguaje, la matemática...sino aquellas emergentes tales como: idiomas, informática /computación, arte, educación física/deporte.

- En casi todas las instituciones educativas se manifiesta la vigencia del modelo constructivista. No obstante, aparecen, por un lado, incongruencias epistemológicasprocedimentales y, por otro se desconocen los resultados alcanzados.

- En casi todos los Centros Educativos se dice conocer la misión institucional. Sin embargo, se presenta una clara contradicción cuando se requiere conocer el contenido de la misma. En lo que se refiere a la Visión existen diversas posiciones. En este contexto, es importante reconocer el hecho de que ya se trabaja con el enfoque de 


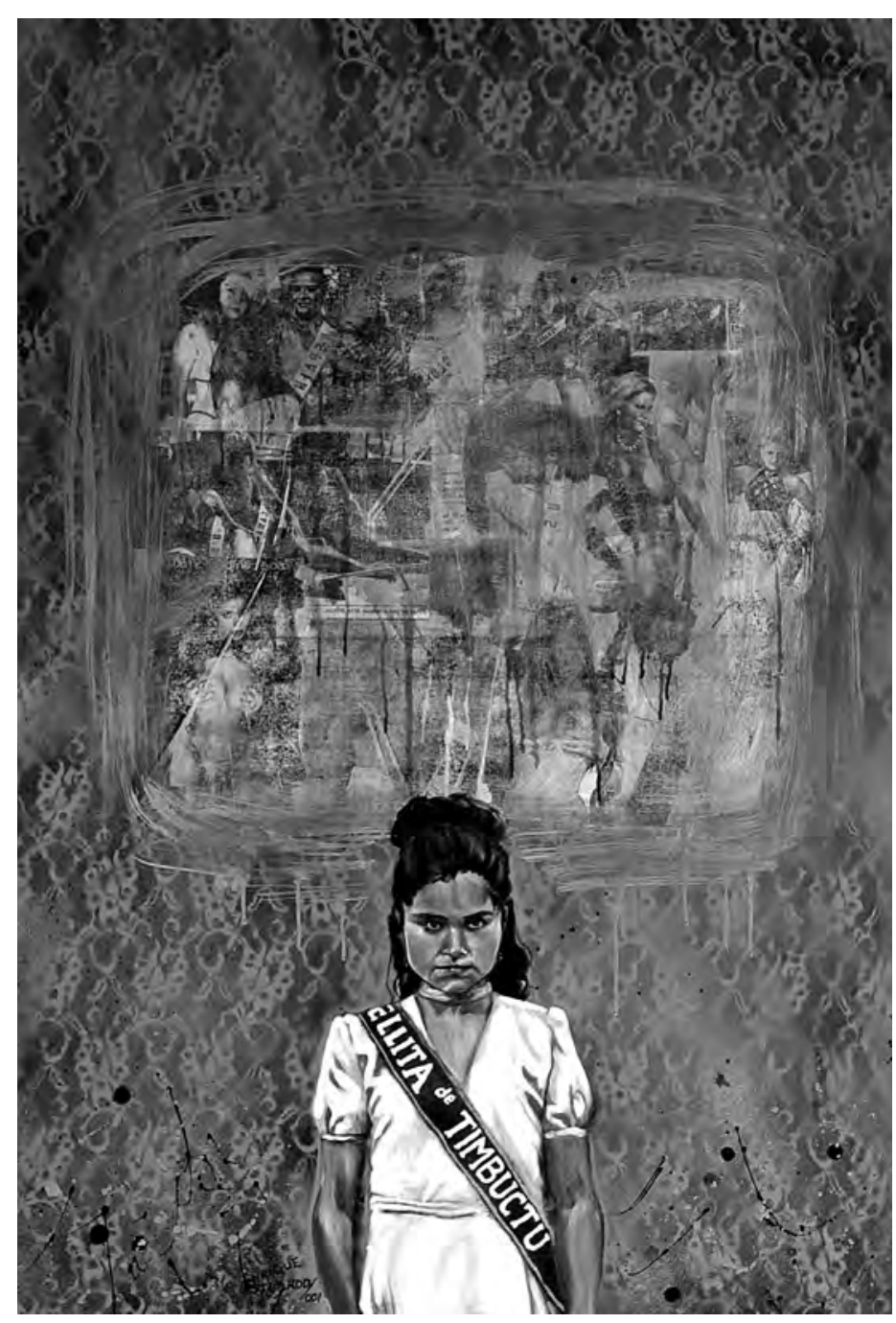

Niña reina. Estampación y acrílico sobre tela. 2001

la planificación estratégica, pero queda en duda la eficacia de la dirección y gestión administrativa del mencionado plan y sus proyectos.

- La gerencia y gestión son los componentes de mayor inquietud en el campo de la administración educativa. Se advierte que existen serias limitaciones de falta de recursos económicos junto a complejos problemas de falta de comunicación y colabo- ración del cuerpo de docentes al interior de las instituciones. Este problema requiere de una oportuna y diligente atención especialmente para los directores y sus colaboradores, presentando alternativas de formación y capacitación.

- El Currículo, es un instrumento de obligada referencia en la educación de los infantes. Sin embargo, la investigación, deja entrever algunas limitaciones y ausencias de carácter teórico, epistemológico, praxológico y metodológico.

- Los recursos didácticos son fundamentales en la construcción del conocimiento y el reforzamiento del aprendizaje. Esto lo conocen los docentes cuando utilizan en su práctica recursos convencionales tales como textos y materiales impresos. Lamentablemente todavía es incipiente el afán por buscar nuevas alternativas con la incorporación de las NTICs, uso de computadores, materiales digitales, multimedia e Internet...

- La estrategia pedagógica más utilizada, en las instituciones investigadas, es la de los juegos didácticos, ya que genera en el niño un deseo por aprender de manera motivada y acogedora. Existen, por otro lado, ciertas experiencias, muy limitadas, que tratan de incursionar en la investigación como un componente básico del proceso de enseñanza-aprendizaje. Por la importancia estratégica de este proceso inicial sería de mucha valía reforzar su crecimiento y desarrollo por parte de la UPS.

- La evaluación educativa es un campo que, desde tiempo atrás presenta serias inquietudes. Hay evidencias claras de que se continúa con enfoques de corte tradicional, es decir, con exámenes y pruebas que tratan de evaluar fundamentalmente conocimientos y en pequeña escala las destrezas adquiridas por los niños. Es una deuda pendiente la evaluación de los diferentes componentes que dicen de la integralidad del desarrollo humano de nuestros niños, 
como mecanismo fundamental de re-información de los procesos educativos. Este es un campo que necesita ser abordado de manera prioritaria.

- En cuanto a la evaluación de la gestión de los docentes es importante reconocer los esfuerzos realizados. Sin embargo necesita ser reforzado este proceso, tomando en consideración los contextos teóricos y epistemológicos de los sistemas de desarrollo del talento humano. En la escuela, la evaluación del desempeño profesional, va ligada al desarrollo cualitativo de la educación y tiene que articularse en un sistema de desarrollo y crecimiento profesional, con el reconocimiento exhaustivo de su gestión, en su multidimensionalidad: social, económica, cultural, administrativa...

- Los resultados de este estudio al constituirse en insumos básicos para la construcción de las Líneas de Investigación de la Carrera de Pedagogía de la UPS-Q., posibilitarán la búsqueda alternativas de solución a los complejos problemas que afronta la gestión docente de la Educación Básica del país, con el concurso y aporte de su cuerpo de docentes, estudiantes y directivos.

- La estructuración de un Sistema de investigación educativan en la Carrera de Pedagogía, permitirá transitar de la docencia profesionalizante hacia una docencia investigativa.

\section{Bibliografía}

- BENVENUNTO, Ángelo: Las tecnologías de Información y Comunicaciones (TIC) en la docencia, Ponencia, Congreso Internacional de Innovación Universitaria. Quito. 2006

- CORTIJO, René: Modelo Curricular por competencias y Proyectos. De los problemas a los productos de

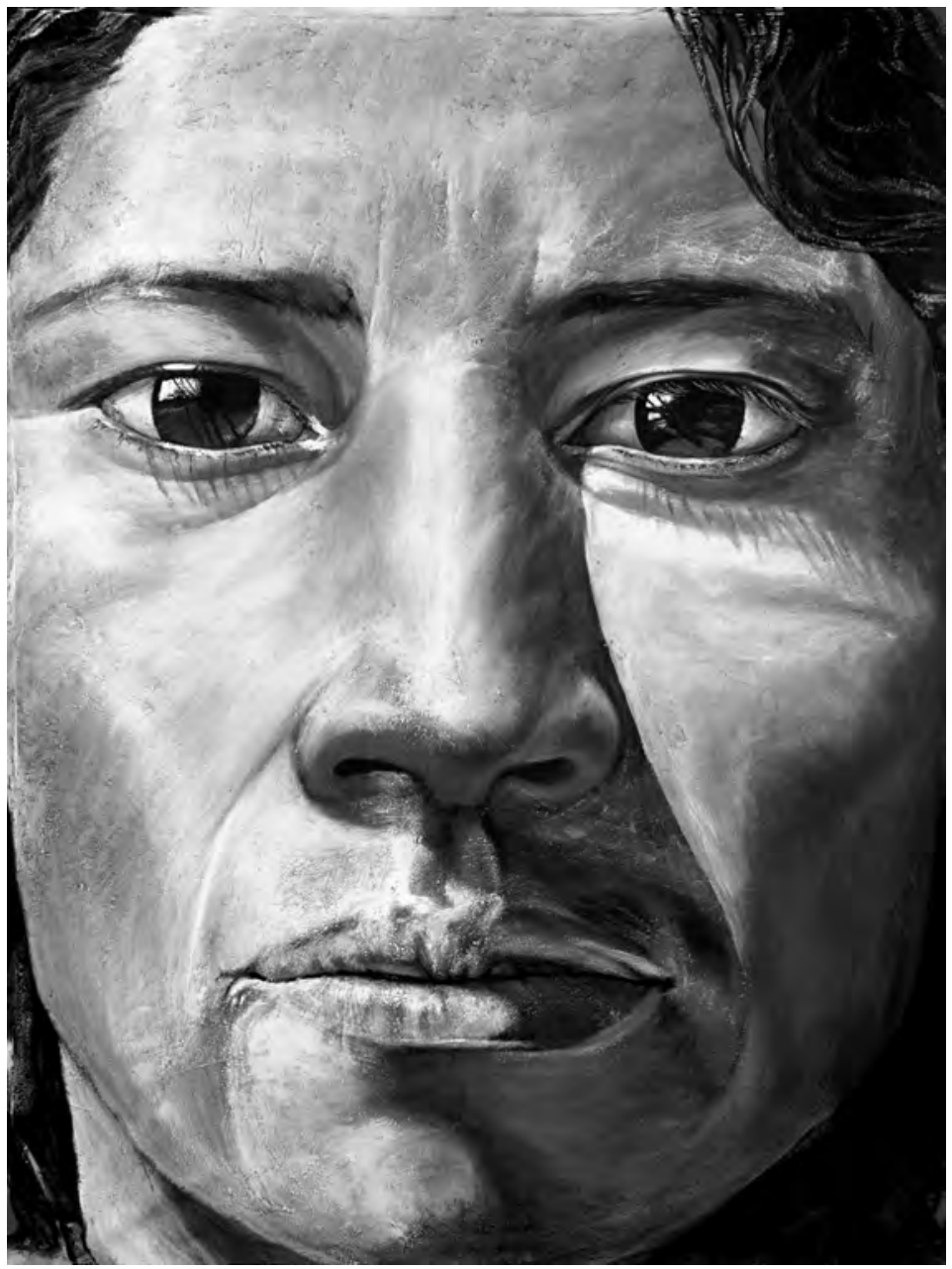

Quilago (32 módulos). Acrílico sobre tela. 2004

aprendizaje. Quito: Klendarios. 2007

- PNUD: Educación: La Agenda del Siglo XXI. Hacia un Desarrollo Humano, Director: Hernando Gómez, PNUD-TM (edit). Colombia. 1998

- ROBAYO, Iván et. al.: Modelo educativo Basado en Competencias con Enfoque Sistémico Prospectivo de la Escuela Politécnica del Ejército, Ponencia, Congreso Internacional de Innovación Universitaria. 2006

- SUÁREZ, Reinaldo: La Educación, México: Trillas. 2002 